\title{
Prescrição e uso de medicamentos por gestantes usuárias do Sistema Único de Saúde
}

\section{Prescription and use of medicines by pregnant users of the Unified Health System}

\author{
Adriana Mestriner Felipe de Meloํㅡ, Edilene Alves dos Santos², \\ Marcos Ávalo Berndt ${ }^{3}$, Luis Arthur Spinola Castilho ${ }^{4}$, \\ Nuccia Garcia Regiani Braga ${ }^{2}$, Kyara Marques Onda ${ }^{2}$
}

\section{Resumo}

\begin{abstract}
Apesar do uso de medicamentos durante a gestação ser um assunto bastante polêmico, existe consenso que o uso desnecessário pode provocar efeitos irreparáveis ao feto. Com o objetivo de descrever o uso de medicamentos prescritos, realizou-se um estudo descritivo em gestantes em pré-natal de três unidades do Sistema Único de Saúde (SUS) no município de Dourados, estado de Mato Grosso do Sul. Os dados sobre o uso de medicamentos foram coletados através de consulta à cópia das prescrições e prontuários das gestantes. Foram analisadas 115 receitas medicamentosas para gestantes atendidas em consultas de rotinas, sendo encontrada a média de 1,4 medicamento por prescrição; sendo $77,8 \%$ prescritos pelo nome genérico, 89 \% constavam na Relação Municipal de Medicamentos Essenciais, enquanto $81 \%$ constavam na Relação Nacional de Medicamentos Essenciais. As preparações antianêmicas foram prescritas em todos os trimestres de gestação, já os analgésicos apareceram numa frequência maior no segundo e terceiro trimestres, bem como os antibacterianos de uso sistêmico. O sulfato ferroso, ácido fólico e paracetamol foram os medicamentos mais prescritos, sendo a maioria pertencente às classes A e B segundo o potencial de risco pela Food and Drug Administration (FDA). A prescrição de medicamentos a gestantes atende aos indicadores de prescrição da Organização Mundial da Saúde (OMS). O uso de medicamentos é frequente e a maioria das gestantes utilizou um ou mais fármacos prescritos durante a gestação.
\end{abstract}

Palavras-chave: Farmacoepidemiologia. Utilização de medicamentos. Gestantes.

\footnotetext{
${ }^{1}$ Doutorado em Ciências da Saúde pela Universidade de Brasília (UnB), Brasília, Distrito Federal, Brasil. Docente do Centro Universitário da Grande Dourados (Unigran), Dourados, Mato Grosso do Sul, Brasil. E-mail: mestriner@unigran.br

${ }^{2}$ Graduação em Farmácia pelo Centro Universitário da Grande Dourados, Dourados, Mato Grosso do Sul, Brasil.

${ }^{3}$ Mestrado em Farmácia pela Universidade Federal de Mato Grosso do Sul (UFMS), Campo Grande, Mato Grosso do Sul, Brasil. Farmacêutico da Prefeitura Municipal de Nova Alvorada do Sul, Mato Grosso do Sul, Brasil.

${ }^{4}$ Mestrado em Ciências da Saúde pela Universidade Federal da Grande Dourados (UFGD), Dourados, Mato Grosso do Sul, Brasil. Docente do Centro Universitário da Grande Dourados, Dourados, Mato Grosso do Sul, Brasil.
} 


\begin{abstract}
Although the use of medications during pregnancy is a very controversial subject, there is a consensus that unnecessary use can cause irreparable effects on the fetus. In order to describe the use of prescription drugs, a descriptive study was carried out on pregnant women in prenatal care at three units of the Unified Health System (SUS) in the city of Dourados, State of Mato Grosso do Sul. use of medicines was collected through consultation with a copy of the prescriptions and medical records of the pregnant women. 115 medication prescriptions for pregnant women attended in routine consultations were analyzed, with an average of 1.4 medications per prescription; $77.8 \%$ of which were prescribed by the generic name, $89 \%$ were on the Municipal List of Essential Medicines, while $81 \%$ were on the National List of Essential Medicines. Anti-anemic preparations were prescribed in all trimesters of pregnancy, while painkillers appeared more frequently in the second and third trimesters, as well as antibacterials for systemic use. Ferrous sulfate, folic acid and paracetamol were the most prescribed drugs, with the majority belonging to classes $\mathrm{A}$ and $\mathrm{B}$ according to the risk potential by the Food and Drug Administration (FDA). The prescription of medications to pregnant women meets the prescription indicators of the World Health Organization (WHO). The use of medications is frequent, and most pregnant women used one or more drugs prescribed during pregnancy.
\end{abstract}

Keywords: Pharmacoepidemiology. Drug utilization. Pregnant women.

\section{Introdução}

O uso de medicamentos durante a gestação, mesmo sendo feito com cautela, ainda é algo frequente, ${ }^{(1-4)}$ seja por influência da mídia, grau de instrução, fatores sociais e econômicos, bem como por possíveis intercorrências que possam ocorrer durante esse período. ${ }^{(5)}$

A Organização Mundial da Saúde (OMS) ${ }^{(6-7)}$ padronizou alguns indicadores relativos à prescrição, à assistência ao paciente ou ainda aos serviços oferecidos, como forma de homogeneizar os parâmetros-chave para descrever a situação de um país, região ou centro de saúde no que se refere ao uso de medicamentos. Dentre os elementos que constituem parâmetros para a análise sobre a utilização de medicamentos padronizados pela OMS estão: média de medicamentos por prescrição médica, porcentagem de medicamentos prescritos pelo nome genérico, porcentagem de medicamentos prescritos da lista de medicamentos essenciais, porcentagem de antibióticos prescritos e porcentagem de injetáveis prescritos.

Para os estudos envolvendo medicamentos administrados durante a gestação, a classificação dos fármacos elaborada pela agência federal norteamericana Food and Drug Administration (FDA) é utilizada e classifica os medicamentos em cinco categorias (A, B, C, D, e X) variando em complexidade crescente de risco. Nesse aspecto, os Estudos de Utilização de Medicamentos (EUM) constituem uma das ferramentas úteis para elucidação e conhecimento desta temática. ${ }^{(8)}$

Desta forma, a utilização de medicamentos por meio de prescrições aviadas a mulheres no período gestacional tem sido alvo de pesquisas em vários países, dentre eles, Índia, ${ }^{(9)}$ Alemanha, ${ }^{(10)}$ Argentina, ${ }^{(11)}$ Eslováquia, ${ }^{(12)}$ Espanha, ${ }^{(13)}$ Sudão ${ }^{(14)}$ e Paquistão, ${ }^{(15)}$ dentre outros locais. No Paquistão, pelo menos um medicamento foi prescrito nas 3.769 receitas aviadas a gestantes atendidas em cinco unidades hospitalares a gestantes, na maioria no terceiro trimestre de gestação, seguido do segundo trimestre. A média de medicamentos prescritos foi de 1,6 por prescrição, enquanto as classes medicamentosas mais prescritas foram suplementos vitamínicos e minerais $(79,4 \%)$, analgésicos $(6,2 \%)$ e antibacterianos $(2,2 \%)$. Um dado preocupante é que $0,8 \%$ das prescrições continha medicações consideradas teratogênicas, como misoprostol, carbimazol ou metotrexato. ${ }^{(15)}$ $\mathrm{Na}$ Argentina, a prescrição de suplementos de ferro aconteceu, principalmente, com finalidades terapêuticas e não como medida preventiva da 
deficiência de ferro. Outro dado interessante foi que a disponibilidade do medicamento não era totalmente contemplada pela rede de atenção primária de saúde em cinco cidades estudadas. ${ }^{(11)}$ $\mathrm{Na}$ Espanha, uma correlação positiva foi encontrada entre o número de antibióticos prescritos e o número de complicações das vias respiratórias e urinárias em pacientes hospitalizados. ${ }^{(13)}$

No Brasil, a utilização de medicamentos por gestantes não é diferente, ${ }^{(16)}$ visto que o uso do ácido fólico, suplementação de ferro e vitaminas é prática frequente durante o pré-natal, também por demonstrar cuidado e adesão ao tratamento e acompanhamento do pré-natal. A média de medicamentos prescritos é de 2,4 medicamentos por prescrição ${ }^{(17)}$ e a perspectiva é que seja utilizado pelo menos um fármaco durante a gravidez. Dentre as classes mais utilizadas estão os antianêmicos $(35,6 \%)$, analgésicos $(24,9 \%)$, drogas para distúrbios gastrintestinais $(9,1 \%)$ e vitaminas $(7 \%){ }^{(17)}$ Apesar do elevado número de medicamentos, mais da metade (70\%) pertencem à classe $\mathrm{A}$ ou $\mathrm{B}$, segundo a classificação de risco pela FDA. Existe uma forte ligação com o grau de instrução e fatores socioeconômicos relacionados a essa utilização de medicamentos durante a gestação.

Considerando a importância e relevância do uso racional de medicamentos durante a gestação, o objetivo desta pesquisa foi avaliar as prescrições de medicamentos a gestantes usuárias do Sistema Único de Saúde (SUS) visando determinar o uso de medicamentos quanto às classes farmacêuticas, aos indicadores de prescrição estabelecidos pela OMS e a classificação de risco adotada pela FDA.

\section{Material e Métodos}

Estudo descritivo, do tipo transversal retrospectivo, no período compreendido entre os meses de outubro de 2009 e fevereiro de $2010 \mathrm{em}$ três unidades básicas de saúde (UBSs) da cidade de Dourados, no estado de Mato Grosso do Sul, Brasil, por terem o programa de acompanhamento ao pré-natal, associadas a farmácias, na unidade. O projeto de pesquisa foi aprovado pelo Comitê de Ética em Pesquisa da Universidade Federal de Mato Grosso do Sul (1491/2009) conforme determinação do Conselho Nacional de Saúde para pesquisas envolvendo seres humanos (Resolução CNS 466\2012).

O estudo foi realizado no município de Dourados - MS, que possuía uma população de 189.762 habitantes no momento da pesquisa. ${ }^{(18)}$ $\mathrm{O}$ atendimento primário do município no momento da pesquisa ocorria em cinco unidades básicas de saúde e outras 43 unidades da Estratégia Saúde da Família (ESF). Os dados sobre os indicadores de prescrição foram pela análise de prescrições obtidas de três unidades por conveniência, considerando que existiam 115 gestantes cadastradas em estudo no período que antecedeu a pesquisa. Foram excluídas aquelas gestantes que apresentavam gestação de risco, indígenas ou que necessitavam atendimento de urgência.

A coleta de dados foi realizada em formulário de entrevista semiestruturado (dados pessoais e trimestre de gestação) e o acesso à cópia carbonada da prescrição possibilitou obter informações sobre a utilização dos medicamentos pelas gestantes: 1) número médio de medicamentos por prescrição; 2) porcentagem de medicamentos prescritos pelo nome genérico; 3) porcentagem de receitas em que se prescrevem antibióticos; 4) porcentagem de medicamentos prescritos que pertencem à Relação Nacional de Medicamentos Essenciais; 5) porcentagem de medicamentos prescritos que pertencem à Relação Municipal de Medicamentos Essencias; e 6) legibilidade da prescrição. Os medicamentos prescritos em cada receita foram listados por seus nomes genéricos e classificados obedecendo à classificação Anatômica, Terapêutica e Química (Anatomical Therapeutic Chemical ATC) da OMS para os estudos de utilização de medicamentos, bem como de acordo com o risco seguindo a padronização da FDA. ${ }^{(19)}$

Os dados foram codificados e digitados no pacote estatístico SPSS $^{\circledR}$ for Windows ${ }^{\circledR}$, versão 15.0. Nesse banco de dados foi possível realizar a identificação e classificação das variáveis investigadas, permitindo a análise descritiva dos dados. 


\section{Resultados e Discussão}

Descrição da população estudada

Foram entrevistadas 115 gestantes após consulta de rotina do programa de pré-natal em três unidades básicas de saúde da cidade de Dourados, Mato Grosso do Sul, envolvidas na pesquisa. As gestantes tinham idade entre $13 \mathrm{e} 44$ anos; a maioria tinha entre 18 e 35 anos. Um dado interessante é que sete das 115 gestantes $(6,1 \%)$ eram menores de idade (Tabela 1).

Tabela 1 - Distribuição das gestantes pela faixa etária, atendidas em três unidades básicas de saúde de Dourados - MS, no período de outubro de 2009 a fevereiro de 2010.

\begin{tabular}{ccc}
\hline Faixa etária (anos) & Frequência absoluta (n) & Frequência relativa (\%) \\
\hline$<18$ & 7 & 6,1 \\
$18-23$ & 36 & 31,3 \\
$24-29$ & 29 & 25,2 \\
$30-35$ & 25 & 21,7 \\
$>35$ & 13 & 11,3 \\
Não informada & 5 & 4,3 \\
Total & 115 & 100 \\
\hline
\end{tabular}

Fonte: Autores

Das 115 gestantes entrevistadas, 15 delas $(13,0 \%)$ estavam no primeiro trimestre de gestação, $45(39,1 \%)$ no segundo trimestre de gestação e $51(44,3 \%)$ no terceiro trimestre de gestação. O trimestre de gestação é um fator relevante para atendimento ao programa de pré-natal, pois quanto mais cedo a gestante aderir ao programa melhor para a mãe e para o bebê, visto que os cuidados necessários devem ser iniciados até 120 dias do início da gestação. Outro fator que reforça esse início precoce de assistência à gestante é que existe uma preocupação maior para gestantes no primeiro trimestre de gestação, pois o risco de másformações é maior nesse período, especialmente quando ocorre exposição a agentes teratogênicos, como os medicamentos pertencentes à classe $\mathrm{X} .{ }^{(20)}$

Uso de medicamentos durante a gravidez

Ao todo foram prescritos 163 medicamentos, entretanto, a média foi de 1,4 medicamento por prescrição (Tabela 2), número inferior à média de 3,3 medicamentos citada pelo Collaborative Group on Drug Use in Pregnancy (CGDUP). ${ }^{(21)}$

Tabela 2 - Avaliação dos indicadores de prescrição de medicamentos após consulta de gestantes em três unidades básicas de saúde de Dourados - MS, atendidas em consultas de rotina do programa de pré-natal, no período de outubro de 2009 a fevereiro de 2010.

\begin{tabular}{lccc}
\hline \multirow{2}{*}{ Indicadores de prescrição } & \multicolumn{2}{c}{ Valores } & \\
\cline { 2 - 3 } & $\mathbf{P}$ & $\mathbf{N}$ & $\%$ \\
\hline Número médio de medicamentos por prescrição. & 115 & 163 & $1,4^{*}$ \\
Número de medicamentos prescritos pelo nome genérico. & 126 & 163 & 77,8 \\
Número de prescritos constantes da lista nacional de medicamentos (RENAME).*** & 132 & 163 & 81,0 \\
Número de prescritos constantes da lista municipal de medicamentos (REMUME).** & 146 & 163 & 89,0 \\
\hline
\end{tabular}

Legenda: P: número de medicamentos relacionados com o indicador referido; N: amostra total de medicamentos. *Não está em média aritmética, assim lê-se 1,4 medicamento por prescrição. **Nome e/ou apresentação.

Fonte: Autores 
A maioria das prescrições estava legível pela percepção das gestantes $(68,7 \%)$. Já com relação aos dados do paciente, o nome estava presente em 96 prescrições $(73,4 \%)$, enquanto o nome do médico, bem como o número do seu registro profissional e assinatura estavam presentes em $72 \%$ das prescrições. O endereço do prescritor foi encontrado em 85 prescrições (72\%). O uso de carimbo que identificasse a unidade, endereço e telefone da Unidade Básica de Saúde foi adotado como suficiente para atender esse indicador.

A associação com o nível de escolaridade foi previamente reportada em estudo realizado por Mengue e colaboradores (2004) ${ }^{(22)}$ envolvendo 5.564 gestantes atendidas pelo programa de prénatal em seis cidades brasileiras no período de 1991 a 1995, que mostrou maior ocorrência do uso de medicamentos multivitamínicos e dos que atuam no sistema digestivo. Osório-de-Castro et al.(4) estudaram o uso de medicamentos em gestantes internadas para o parto por meio de prontuários e um achado alarmante foi que algumas praticaram automedicação com misoprostol, durante a gestação, com finalidade abortiva. O que chama atenção é que essa medicação não é mais de venda livre há pelo menos dez anos. Na cidade de Pelotas, estado do Rio Grande do Sul, das 1.450 mulheres internadas em cinco maternidades, a prevalência do uso de ácido fólico na gestação foi maior do que comparado com o uso no período periconcepcional. ${ }^{(22)}$

As preparações antianêmicas foram prescritas em todos os trimestres de gestação, já os analgésicos apareceram numa frequência maior no segundo e terceiro trimestres, bem como os antibacterianos de uso sistêmico. A maioria dos medicamentos prescritos (126) estava pelo nome genérico e fazia parte da Relação Nacional e da Municipal de Medicamentos Essenciais (RENAME e REMUME). Desta forma, o percentual foi considerado satisfatório, visto que a Lei 9787, de 10 de fevereiro de 1999, ${ }^{(23)}$ define a obrigatoriedade dos serviços públicos em prescreverem a totalidade dos medicamentos pelo nome genérico. Entretanto, os dados referentes à posologia estavam presentes em apenas 16 medicamentos. Considerando que o uso correto de medicamentos pode estar diretamente relacionado às informações corretas disponíveis na prescrição associada a uma orientação farmacêutica adequada, os dados como forma farmacêutica e via de administração contemplavam esses preceitos. Contudo, no que diz respeito à posologia, algumas informações ainda mostram-se incompletas ou deficientes, o que poderia acarretar danos no que diz respeito ao Uso Racional de Medicamentos (URM) (Tabela 3).

Tabela 3 - Presença de dados referentes aos medicamentos prescritos a gestantes após consulta de rotina em três unidades básicas de saúde (UBSs) de Dourados - MS, no período de outubro de 2009 a fevereiro de 2010.

\begin{tabular}{lcc}
\hline Informação & Frequência absoluta (n) & Frequência relativa (\%) \\
\hline Forma farmacêutica & 98 & 60,1 \\
Posologia completa & 16 & 9,9 \\
- Dose & 33 & 20,2 \\
- Intervalo & 108 & 66,3 \\
- Duração & 56 & 34,4 \\
Vias de administração & 94 & 57,7 \\
\hline
\end{tabular}

Fonte: Autores

Assim, os indicadores de prescrição da OMS foram úteis para conhecer o perfil da prescrição medicamentosa e comparação com outros serviços, possibilitando uma possível intervenção na saúde pública do município de Dourados - MS, visando proporcionar uso seguro e correto de medicamentos 
para gestantes. As classes de medicamentos mais prescritos foram preparações antianêmicas (V03) $(65,42 \%$,$) , seguido dos analgésicos (N02) (27,17 \%)$, antibacterianos de uso sistêmico (J01) (15, 9,5\%), antieméticos/antinauseantes (A04) e medicamentos para distúrbios relacionados com ácidos (A02), ambos com 5\% cada um (8) (Tabela 4). De Acordo com Carmo e Nitrine, ${ }^{(24)}$ os anti-infeciosos são os grupos farmacológicos mais prescritos no primeiro trimestre de gestação. Os antianêmicos e analgésicos também foram as duas classes de medicamentos mais prescritos no estudo realizado por Guerra et al., ${ }^{(17)}$ fato este relacionado aos eventos frequentes na gravidez, como náusea e vômitos.

Tabela 4 - Distribuição dos medicamentos prescritos para gestantes em consultas de rotina do pré-natal em três unidades básicas de saúde de Dourados - MS, segundo a classificação Anatomical Therapeutic Chemical (ATC), no período de outubro de 2009 a fevereiro de 2010.

\begin{tabular}{clcc}
\hline \multirow{2}{*}{ ATC* } & \multicolumn{1}{c}{ Classe de medicamentos } & \multicolumn{2}{c}{ Frequência } \\
\cline { 3 - 4 } & & Absoluta (n) & Relativa (\%) \\
\hline A02 & Medicação distúrbios relacionados com ácidos & 8 & 4,91 \\
A03 & Medicação distúrbios funcionais do trato intestinal & 3 & 1,84 \\
A04 & Antianêmicos e antinauseantes & 8 & 4,91 \\
A11 & Vitaminas & 1 & 0,62 \\
B03 & Preparações antianêmicas & 65 & 39,9 \\
C01 & Heterosídeos cardíacos & 1 & 0,62 \\
C04 & Vasodilatadores periféricos & 2 & 1,20 \\
D07 & Preparações dermatológicas com corticoides & 3 & 1,84 \\
G01 & Anti-infecciosos e antissépticos ginecológicos & 9 & 5,50 \\
G03 & Hormônios sexuais moduladores do sistema genital & 1 & 0,62 \\
J01 & Antibacterianos de uso sistêmico & 15 & 9,20 \\
J02 & Antimicóticos de uso sistêmicos & 1 & 0,62 \\
J05 & Antivirais de uso sistêmico & 1 & 0,62 \\
M01 & Anti-inflamatório e antirreumático & 1 & 0,62 \\
N02 & Analgésicos & 27 & 16,60 \\
R03 & Medicações para doenças obstrutivas de vias aéreas & 3 & 1,84 \\
R05 & Preparações para tosse & 2 & 1,20 \\
R06 & Anti-histamínicos de uso sistêmico & 4 & 2,50 \\
S01 & Oftalmológicos & 1 & 0,62 \\
$* *$ & Não identificados & 7 & 4,30 \\
Total & & 163 & 100,0 \\
\hline
\end{tabular}

Legenda: *segundo subgrupo terapêutico; **não identificados.

Fonte: Autores

A maioria dos medicamentos prescritos pertence às classes $\mathrm{A}$ e $\mathrm{B}$ segundo a classificação da agência FDA (sulfato ferroso; acido fólico; paracetamol; cefalexina; ambroxol e salbutamol) (Tabela 5). Do total de medicamentos prescritos, aproximadamente $13 \%$ foram classificados na categoria $\mathrm{C}$ - por exemplo, o butilbrometo de escopolamina. É importante destacar que os medicamentos pertencentes a essa categoria devem ser utilizados com cautela, pois seu uso é aceito em gestantes quando os benefícios forem justificados, visto que seus efeitos deletérios ao feto não foram evidenciados em experimentos em animais, mas precisam ser considerados. 
A prevalência de más-formações congênitas parece ter sido relacionada ao consumo elevado de medicamentos pertencentes à classe $\mathrm{X} \cdot{ }^{(20)}$ Cabe salientar que os resultados apresentados como sendo de consumo de medicamentos são na verdade estimando sua utilização a partir da prescrição medicamentosa. Desta forma, a pesquisa possui o viés daqueles medicamentos que apesar de serem prescritos podem não terem sido consumidos pelas gestantes por diferentes fatores. Vale destacar que a utilização dos medicamentos foi embasada na prescrição e não de fato na pesquisa de uso por elas. De qualquer forma, apesar dessa limitação, acredita-se que os resultados obtidos poderão ser úteis para o serviço de assistência farmacêutica do município, visto que a avaliação das preferências dos prescritores pode ser fundamental para a tomada de decisões sobre políticas de promoção do URM. Vale salientar que, segundo a teoria econômica, para conhecer as preferências das pessoas pela observação do comportamento, tem-se de pressupor que suas preferências permanecerão imutáveis durante um período de tempo acima de um mês. Essa condição é válida para a prescrição de medicamentos, uma vez que não parece provável que as preferências de um determinado prescritor sofram mudanças significativas em um curto espaço de tempo. ${ }^{(25)}$

Tabela 5 - Distribuição dos medicamentos prescritos para gestantes em consultas de rotina do pré-natal em três unidades básicas de saúde (UBSs) de Dourados - MS, de acordo com a classificação de risco da Food and Drug Administration (FDA).

\begin{tabular}{|c|c|c|c|}
\hline \multirow{2}{*}{ Risco } & \multirow{2}{*}{ Medicamentos } & \multicolumn{2}{|c|}{ Frequência } \\
\hline & & Absoluta (n) & Relativa (\%) \\
\hline A & Ácido fólico, sulfato ferroso. & 65 & 39,9 \\
\hline B & $\begin{array}{l}\text { Ambroxol, amoxicilina, azitromicina, cefalexina, complexo B, } \\
\text { dexclordifeniramina, diclofenaco, dipirona, metoclopramida, } \\
\text { loratadina, metronidazol, nistatina, paracetamol, ranitidina, } \\
\text { salbutamol. }\end{array}$ & 61 & 37,1 \\
\hline $\mathrm{C}$ & $\begin{array}{l}\text { Cetoconazol, utilbrometo de escopolamina, butilbrometo de } \\
\text { escopolamina + dipirona sódica, isoxsuprina, dexametasona, } \\
\text { dexametasona + sulfato de neomicina + sulfato de polimixina B, } \\
\text { dexametasona + neomicina, dimeticona, fluconazol, omeprazol, } \\
\text { hidróxido de alumínio. }\end{array}$ & 22 & 13,3 \\
\hline $\mathrm{D}$ & Progesterona, tetraciclina, zidovudina. & 3 & 1,8 \\
\hline NI & $\begin{array}{l}\text { Cloridrato de benzidamina, dimenidrinato, lanolina, miconazol, } \\
\text { vitamina } \mathrm{C}+\text { piperidolato }+ \text { hesperidina-complexo, dipropionato } \\
\text { de betametasona }+ \text { cetoconazol. }\end{array}$ & 11 & 6,6 \\
\hline $\mathrm{X}$ & Etilefrina. & 1 & 0,6 \\
\hline
\end{tabular}

Legenda: (A) categoria A: estão os medicamentos que em estudos controlados em mulheres não demonstram risco para o feto; (B) categoria $\mathrm{B}$ : aqueles em que os estudos com animais não demonstraram risco fetal no primeiro trimestre; mas que também não existem estudos em mulheres gestantes, ou que mostraram risco em experimentação animal que não foram confirmados em humanos; $(\mathrm{C})$ categoria $\mathrm{C}$ : em experimentação animal mostraram riscos para o feto, mas que não existem estudos em mulheres, ou ainda, aqueles que não depõem de estudos tanto em animais quanto em mulheres. Nessa categoria, a indicação terapêutica poderá ser justificada quando o benefício justificar o risco para o feto; (D) categoria D: estão aqueles com evidências positivas de risco fetal humano, porém os benefícios potenciais para a mulher grávida podem, eventualmente, justificar seu uso; (X) categoria X: inclui os medicamentos contraindicados na gestação, pois estudos em animais e em mulheres grávidas demonstraram evidência clara de risco fetal; (NI): risco não conhecido.

Fonte: Autores 
Em São Paulo - SP, a média de medicamentos utilizados por 47 gestantes atendidas em uma Unidade de Saúde da Família (USF) foi de 3,6 por prescrição. O suplemento de ferro esteve presente em todas as prescrições, enquanto a classe dos antibióticos foi a segunda classe de medicamentos mais prescrita $(78,7 \%){ }^{(26)}$

Já em Piracicaba - SP, 44\% de um total de 427 gestantes consultadas durante o pré-natal receberam prescrição médica contendo pelo menos um medicamento. As classes farmacológicas dos medicamentos mais prescritos no primeiro trimestre de gestação foram as que atuam no sistema hematopoiético (37,5\%), sistema digestivo $(26,1 \%)$ e anti-infecciosos (18,2\%). Dos medicamentos prescritos, praticamente a metade atendia à recomendação da OMS quanto aos indicadores de prescrição e serviço, respectivamente, com a prescrição pelo nome genérico e pela disponibilidade na rede pública para acesso da população, constando na RENAME e na REMUME. ${ }^{(24)}$

Erebara et $a l .{ }^{(27)}$ destacam a importância de conscientizar as gestantes sobre os efeitos nocivos do uso irracional de analgésicos, descongestionantes e anti-histamínicos nos quadros de resfriados. Isso porque, apesar de serem medicamentos de venda livre, precisam ser administrados com cautela, especialmente durante a gravidez. Muitas mulheres gestantes não sabem do risco que estão correndo ao tomar uma medicação em horários diferentes daqueles prescritos pelo médico, não têm conhecimento da gravidade do problema e das futuras consequências que podem surgir ao se tomar uma medicação por conta própria, não sabem que o feto pode ter alterações, má-formação, ou vindo ocorrer até mesmo a morte de ambos.

Como bem lembrado por Osório-de-Castro et al., ${ }^{(4)}$ embora a tragédia da talidomida tenha marcado o início da reflexão sobre a ocorrência de efeitos adversos de medicamentos usados durante a gestação, as percepções dos prescritores, no âmbito da terapêutica medicamentosa na gravidez, ainda oscilam entre a certeza de que tudo é nocivo e a relativa crença de que tudo é seguro até que se prove o contrário.
Brum et al. ${ }^{(28)}$ encontraram que a prevalência do uso de medicamentos prescritos a gestantes do município de Santa Rosa, Rio Grande do Sul, foi de $90 \%$, correspondendo a uma média de uso de 4,1 medicamentos por gestante, dos quais $83,6 \%$ foram prescritos e 16,4\% foram utilizados por automedicação. Do total de medicamentos, 17,5\% estão incluídos na categoria $\mathrm{C}$ de risco ao feto. $\mathrm{O}$ uso de medicamentos é frequente, e a maioria das gestantes utilizou um ou mais fármacos, prescritos ou não. Esses dados sugerem a necessidade de medidas de intervenção para promover o uso racional de medicamentos durante a gestação.

Já o estudo de Mengue et al. ${ }^{(29)}$ envolvendo 5.564 gestantes atendidas pelo programa de prénatal em seis cidades brasileiras no período de 1991 a 1995 revelou que o uso de medicamentos multivitamínicos e daqueles que atuam no sistema digestivo, prescritos ou não, foi maior em mulheres com maior tempo de escolaridade. Estudo realizado com 887 primigestas no município de Rio Branco, no Acre, demonstrou que a média do uso de medicamentos foi 2,42 e os medicamentos mais consumidos foram os antianêmicos $(47,5 \%)$, suplementos e vitaminas (18,7\%), analgésicos $(13,8 \%)$ e antibióticos $(10,5 \%)$. Na categorização de risco, 69,3\% pertenciam à categoria $\mathrm{A}$; $22,3 \%$ à $\mathrm{B} ; 7,6 \%$ à $\mathrm{C}$ e $0,8 \%$ pertencia à $\mathrm{D}$. Os medicamentos essenciais foram menos utilizados entre as mulheres com maior renda familiar e que realizaram o pré-natal na rede privada. ${ }^{(30)}$

\section{Considerações finais}

As prescrições de medicamentos aviadas para gestantes atendidas em três unidades básicas de saúde de Dourados - MS, atendem aos indicadores de prescrição utilizados para refletir o Uso Racional de Medicamentos pela OMS.

Os medicamentos mais prescritos para gestantes atendidas durante as consultas de rotina do pré-natal em três unidades básicas de saúde do município de Dourados no período de outubro de 2009 a fevereiro de 2010 foram: sulfato ferroso, ácido fólico e paracetamol. 
A maioria dos medicamentos prescritos não oferece risco potencial às gestantes, pois pertence às classes $\mathrm{A}$ e $\mathrm{B}$ segundo a classificação de risco adotada pela FDA.

\section{Referências}

1 Costa DB, Coelho HLL, Santos DB. Utilização de medicamentos antes e durante a gestação: prevalência e fatores associados. Cad. Saúde Pública[Internet].2017[citado2017mar9];33(2): e00126215. Disponível em http://www.scielo. br/scielo.php?script $=$ sci_arttext\&pid=S0102311X2017000205005\&lng=en

2 Ferreira MBC, Wannmacher L, Osório-deCastro, CGS. Fatores capazes de modificar a resposta de fármacos. In: Fuchs FD, Wannmacher L, Ferreira MBC. Farmacologia clínica: fundamentos da terapêutica racional. $3 \mathrm{a}$ ed. Rio de Janeiro: Guanabara Koogan; 2006. p. 63-66.

3 Mendes AMD, Figueiredo CP. Drug use during pregnancy: dermatological approach. An Bras Dermatol. 2000;75(1):87-92.

4 Osorio-de-Castro CGS, Pepe VLE, Luiza VL, Cosendey MAE, Freitas AM, Miranda FF, et al. Uso indicado e uso referido de medicamentos durante a gravidez. Cad. Saúde Pública [Internet]. 2004 [citado 2020 mar 23];20(Suppl 1):S73-S82. Disponível em: http://www.scielo. br/scielo.php?script $=$ sci_arttext\&pid $=\mathrm{S} 0102$ $311 \times 2004000700008 \& \operatorname{lng}=$ en

5 Gomes KRO, Moron AF, Souza e Silva R, Siqueira AAF. Prevalência do uso de medicamentos na gravidez e relações com as características maternas. Rev. Saúde Pública [Internet]. 1999 June [citado 2020 mar 23];33(3): 246-54. Disponível em: http://www.scielo. br/scielo.php?script $=$ sci_arttext\&pid=S0034$89101999000300005 \& \operatorname{lng}=$ en

6 Organização Mundial da Saúde. Using indicators to measure country pharmaceutical situations [Internet]. Geneva: OMS, 2006. [cited 2008 Apr 5]. Available from: http://www.who.int/ medicines/publications/WHOTCM2006.2A.pdf
7 Organização Mundial da Saúde. Guia para boa prescrição médica. Porto Alegre: Artmed; 1998.

8 Romano-Lieber NS. Farmacoepidemiologia. In: Cordeiro $\mathrm{BC}$, Leite $\mathrm{SN}$, organizadores. $\mathrm{O}$ farmacêutico na atenção à saúde. Itajaí: Univali, 2005. p. 113-39.

9 Mathai E, Thomas RJ, Chandy S, Mathai M, Bergstrom S. Antimicrobials for the treatment of urinary tract infection in pregnancy: practices in southern India. Pharmacoepidemiol Drug Saf. 2004;13(9):645-52.

10 Egen-lape V, Hasford J. Drug prescription in pregnancy: analysis of a large statury sickness fund population. Eur J Clin Pharmacol [Internet]. 2004 Nov [cited 2020 Mar 1];60(9):659-66. Available from: http://www. ncbi.nlm.nih.gov/entrez/utils/fref.fcgi?PrId= 3055\&itool=AbstractPlusdef\&uid $=15480609$ $\& \mathrm{db}=$ pubmed\&url=http://dx.doi.org/10.1007/ s00228-004-0817-1

11 Bernztein R, Drake I. Underprescription of iron and clinical practice variability at the primary care level of public medical attention in Argentina. Arch Argent Pediatr. 2008;106(4): 320-7. doi 10.1590/S032500752008000400007 .

12 Durisova A, Magulova L. Drug use-problem in pregnancy. Bratisl Lek Listy. 2004;105(3):123124.

13 Urrusuno RF, González MP, Rojas MAT. Antibiotic prescribing patterns and hospital admissions with respiratory...and urinary tract infections. Eur J Clin Pharmacol. 2008; 64(10):1005-11.

14 Awad AI, Eltayeb IB, Baraka OZ. Changing antibiotics prescribing practices in health centers of Khartoum State, Sudan. Eur J Clin Pharmacol. 2006;62(2):135.

15 Rohra DK, Das N, Azam SI, Solangi NA, Memon Z, Shaikh AM, et al. Drug-prescribing patterns during pregnancy in the tertiary care hospitals of Pakistan: a cross sectional study. BMC pregnancy and childbirth. 2008;8(4):1-5. 
16 Lunet N, Rodrigues $\mathrm{T}$, Correia S, Barros $\mathrm{H}$. Adequacy of prenatal care as a major determinant of folic acid, iron, and vitamin intake during pregnancy. Cad. Saúde Pública [Internet]. 2008 [cited 2020 Mar 2];24(5): 1151-7. Available from: http://www.scielo. br/scielo.php?script=sci_arttext\&pid=S0102$311 X 2008000500022 \& \operatorname{lng}=$ en

17 Guerra GCB, Silva AQB, França LB, Assunção PMC, Cabral RX, Ferreira AAA. Utilização de medicamentos durante a gravidez na cidade de Natal, Rio Grande do Norte, Brasil. Rev Bras Ginecol Obstet. 2008 jan;30(1):12-8.

18 Instituto Brasileiro de Geografia e Estatística IBGE. [Internet]. 2009. [citado 2010 fev. 11]. Disponível em http://www.ibge.gov.br

19 Fuchs FD, Wannmacher L, Ferreira MBC. Farmacologia clínica: fundamentos da terapêutica racional. 3a ed. Rio de Janeiro: Guanabara Koogan; 2006.

20 Ramos WLP, Benevento CE, Malgarin J, Stefanello TF, Ramos ERP. Análise do uso de medicamentos durante a gestação em mães de pacientes portadores de malformações fetais. Saúde e Pesqui. 2008;1(1):59-64.

21 Collaborative Group on Drug Use in Pregnancy (CGDUP). Medication during pregnancy: an intercontinental cooperative study Int J Gynaecol Obstet. 1992;39(3):185-96.

22 Mezzomo CLS, Garcias GL, Sclowitz ML, Sclowitz IT, Brum CB, Fontana T, et al. Prevenção de defeitos do tubo neural: prevalência do uso da suplementação de ácido fólico e fatores associados em gestantes na cidade de Pelotas, Rio Grande do Sul, Brasil. Cad. Saúde Pública. 2007 nov;23(11):27162726.

23 Brasil. Lei 9787 de 10 de fevereiro de 1999. Altera a lei $\mathrm{n}^{\circ} 6360$ de 23 de setembro de 1976, que dispõe sobre a vigilância sanitária, estabelece o medicamento genérico, dispõe sobre a utilização de nomes genéricos em produtos farmacêuticos e dá outras providências. Brasília (DF): Diário Oficial da União; 11 fev 1999. Seção 1, p. 1.
24 Carmo TA, Nitrini SMM. O. Prescrições de medicamentos para gestantes: um estudo farmacoepidemiológico. Cad Saúde Pública. 2004;20(4):1004-13.

25 Mota DM, Silva MGC, Sudo EC, Ortún V. Uso racional de medicamentos: uma abordagem econômica para tomada de decisões. Ciênc Saúde Coletiva. 2008 abr;13(Suppl):589-601.

26 Maeda ST, Secoli SR. Use and cost of medication in low risk pregnant women. Rev. Latino-Am. Enfermagem. 2008;16(2):266-71.

27 Erebara A, Bozzo P, Einarson A, Koren G. Treating the common cold during pregnancy. Can. Fam. Physician. 2008; 54: 687-689.

28 Brum LFS, Pereira P, Felicetti LL, Silveira RD. Utilização de medicamentos por gestantes usuárias do Sistema Único de Saúde no município de Santa Rosa (RS, Brasil). Ciênc. Saúde Coletiva. 2011 maio;16(5):2435-42.

29 Mengue SS, Schenkel EP, Schmidt MI, Duncan BB. Fatores associados ao uso de medicamentos durante a gestação em seis cidades brasileiras. Cad. Saúde Pública. 2004; 20(6):1602-8.

30 Andrade AM, Ramalho AA, Koifman RJ, Dotto LMG, Cunha MA, Opitz SP. Fatores associados ao uso de medicamentos na gestação em primigestas no Município de Rio Branco, Acre, Brasil. Cad. Saúde Pública. 2014 maio; 30(5):1042-56. 